\title{
Avaliação de géis transdérmicos na veiculação da terapia da reposição hormonal
}

\author{
Evaluation of transdermal gels in the delivery of hormone replacement therapy \\ Evaluación de geles transdérmicos en la administración de terapia de reemplazo hormonal
}

Recebido: 25/11/2021 | Revisado: 02/12/2021 | Aceito: 06/12/2021 | Publicado: 16/12/2021

\author{
Stephany de Melo Mesquita \\ ORCID: https://orcid.org/0000-0002-9809-8303 \\ Universidade Federal do Rio de Janeiro, Brasil \\ E-mail: stephanymesquita20@gmail.com \\ Zaida Maria Faria de Freitas \\ ORCID: https://orcid.org/0000-0001-6044-1711 \\ Universidade Federal do Rio de Janeiro, Brasil \\ E-mail: zaidafarmacia@gmail.com \\ Mariana Sato de Souza Bustamante Monteiro \\ ORCID: https://orcid.org/0000-0002-2422-8048 \\ Universidade Federal do Rio de Janeiro, Brasil \\ E-mail: marianasato@pharma.ufrj.br
}

\begin{abstract}
Resumo
A terapia de reposição hormonal (TRH) tem sido cada vez mais utilizada em pessoas que possuem baixos níveis hormonais. Em mulheres menopausais, a terapia é bem conhecida pela associação de estrogênios e progestágenos, enquanto que em homens hipogonadais a terapia é realizada pela reposição da testosterona. Para uma administração mais segura desses hormônios, a via transdérmica tem sido utilizada por promover uma liberação modificada do ativo, além de evitar o efeito de primeira passagem dos fármacos. Entretanto, muito se discute sobre a eficácia das formulações transdérmicas em alcançar os níveis séricos fisiológicos hormonais compatíveis com os níveis terapêuticos. O objetivo do estudo foi realizar uma revisão na literatura sobre a segurança e a eficácia dos géis transdérmicos na veiculação dos hormônios esteroides sexuais: estrogênio, progesterona e testosterona, utilizados na TRH. Foram utilizadas as seguintes plataformas: Google Acadêmico, Pubmed e Periódicos Capes. O uso da via transdérmica para a formulação de géis na veiculação da testosterona alcançou eficácia, porém a segurança não foi garantida pela instabilidade dos níveis séricos hormonais na circulação sistêmica, ao passo que a progesterona não alcançou eficácia, pois não foi capaz de atingir os níveis fisiológicos ideais, enquanto que o estrogênio foi bem tolerável e eficaz sendo a melhor alternativa comparável a formulações orais em eficácia, e mais seguras ao paciente submetido à TRH.
\end{abstract}

Palavras-chave: Estrogênio; Progesterona; Testosterona; Transdérmico.

\begin{abstract}
Hormone replacement therapy (HRT) has been increasingly used in people who have low hormone levels. In menopausal women, the therapy is well known for the association of estrogens and progestin's, while in hypogonadal men, the therapy is carried out through testosterone replacement. For a safer administration of these hormones, the transdermal route has been used to promote a modified release of the active, in addition to avoiding the first-pass effect of the drugs. However, there is much debate about the effectiveness of transdermal formulations in achieving physiological hormonal serum levels compatible with therapeutic levels. The study aimed to conduct a literature review on the safety and efficacy of transdermal gels in the delivery of sex steroid hormones: estrogen, progesterone, and testosterone, used in HRT. The following platforms were used: Academic Google, PubMed, and Capes Periodicals. The use of the transdermal route for the formulation of gels in the delivery of testosterone achieved efficacy, but safety was not guaranteed by the instability of serum hormone levels in the systemic circulation, while progesterone was not effective, as it was not able to reach ideal physiological levels, while estrogen was well tolerable and effective, being the best alternative compared to oral formulations in efficacy and safer for the patient undergoing HRT.
\end{abstract}

Keywords: Estrogen; Progesterone; Testosterone; Transdermal.

\section{Resumen}

La terapia de reemplazo hormonal (TRH) se ha utilizado cada vez más en personas que tienen niveles hormonales bajos. En mujeres menopáusicas, la terapia es bien conocida por la asociación de estrógenos y progestágenos, mientras que en hombres con hipogonadismo, la terapia se lleva a cabo mediante reemplazo de testosterona. Para una administración más segura de estas hormonas, se ha utilizado la vía transdérmica para promover una liberación modificada del activo, además de evitar el efecto de primer paso de los fármacos. Sin embargo, existe un gran debate 
sobre la eficacia de las formulaciones transdérmicas en el logro de niveles séricos hormonales fisiológicos compatibles con los niveles terapéuticos. El estudio tuvo como objetivo realizar una revisión de la literatura sobre la seguridad y eficacia de los geles transdérmicos en la administración de hormonas esteroides sexuales: estrógeno, progesterona y testosterona, utilizadas en la TRH. Se utilizaron las siguientes plataformas: Academic Google, PubMed y Capes Periodicals. El uso de la vía transdérmica para la formulación de geles en la liberación de testosterona logró eficacia, pero la seguridad no estaba garantizada por la inestabilidad de los niveles séricos de hormonas en la circulación sistémica, mientras que la progesterona no fue efectiva, ya que no pudo alcanzar el nivel ideal. niveles fisiológicos, siendo la mejor alternativa frente a las formulaciones orales en eficacia y más segura para el paciente sin TRH.

Palabras clave: Estrógeno; Progesterona; Testosterona; Transdérmica.

\section{Introdução}

A terapia de reposição hormonal (TRH), desde 1960, tem sido muito discutida acerca dos seus riscos e benefícios (Pardini, 2014). A TRH é muito utilizada para tratar os sintomas da menopausa e andropausa, que são caracterizados pela queda da produção dos hormônios sexuais femininos e masculinos, processos naturais de envelhecimento do organismo (Polonini et al., 2011).

O estrogênio é o principal hormônio feminino, cuja ação está relacionada com o controle da ovulação e com o desenvolvimento de características femininas. Em mulheres pode-se destacar o estradiol (E2), que exerce atividade hormonal estrogênica. A sua biossíntese ocorre a partir do colesterol, sendo produzido principalmente pelos folículos, corpo lúteo e placenta, enquanto nos homens o E2 é produzido nos testículos (Kumar et al., 2018). A progesterona é um hormônio sexual esteroide produzido a partir da puberdade pelo corpo lúteo, e pela placenta durante a gravidez. Nas gônadas é sintetizado após a liberação do estrogênio, o qual estimula a liberação do folículo e logo após a sua degradação se transforma em corpo lúteo designado a proteger o embrião, e facilitar sua implantação no útero. A progesterona produzida a partir das gônadas é transportada principalmente no sangue para exercer sua função biológica, enquanto que a progesterona de origem adrenal é amplamente convertida em glicocorticóides e andrógenos (Taraborrelli, 2015; Piette, 2018). A testosterona é o principal hormônio esteróide androgênico produzido pelas células de Leydig nos testículos. É capaz de regular uma série de funções nos homens, como a produção de esperma, desejo sexual, atua por ação direta em diferentes tipos celulares, promove o crescimento e rigidez óssea, aumento da produção de eritrócitos, regula o metabolismo de lipoproteínas, além de atuar como anabolizante proteico nos músculos (Cunha, 2020).

A menopausa causa a progressiva redução da atividade ovariana, a qual é o responsável pela secreção dos hormônios femininos, como o estradiol, a estrona e o estriol. Esta redução hormonal pode ocorrer de forma abrupta e intensa, e levar a alguns sintomas clínicos observados em decorrência do hipoestrogenismo, como alterações tróficas da pele e da mucosa vaginal, ressecamento da mucosa vaginal; tendência a fraturas osteoporóticas pela perda acelerada de massa óssea; distúrbios psicológicos como perda de libido e insônia; modificação do perfil lipídico, como o aumento nos níveis de triglicérides e de lipoproteína de baixa densidade (LDL) e redução nos níveis de proteína de alta densidade (HDL); e fogachos, compreendidos como as ondas repentinas de calor que passam pelo corpo. O conjunto formado por estas mudanças biológicas constitui o que se chama de climatério, isto é, a fase de transição entre a fase reprodutiva e a não reprodutiva nas mulheres devido ao esgotamento dos folículos ovarianos e o consequente declínio da produção de estrógenos resultando a menopausa (Johansen et al., 2020; Melo et al., 2021).

A ausência ou baixa produção de testosterona, nos homens, leva a síndrome clínica conhecida como hipogonadismo. Dentre as causas mais comuns do hipogonadismo, destaca-se o processo natural de envelhecimento, tendo em vista que essa deficiência tende a aumentar com a idade. Os sintomas decorrentes dos baixos níveis de testosterona circulante dependem da idade do paciente, uma vez que a testosterona e seu metabólito ativo têm papel fundamental no início do processo de puberdade. De forma geral, os baixos níveis de androgênios circulantes podem causar distúrbios no desenvolvimento sexual 
masculino, podem levar a redução da fertilidade, disfunção sexual, declínio da força muscular, menor mineralização óssea, distúrbio do metabolismo lipídico e disfunção cognitiva. Sendo assim, tanto a testosterona quanto os seus derivados sintéticos têm sido utilizados no tratamento do hipogonadismo masculino e suas complicações (Cunha, 2020).

A TRH é utilizada, em mulheres e homens, para reduzir os sintomas menopausais e do hipogonadismo, respectivamente. Nos anos 2000, surgiu um novo protocolo para a utilização da TRH que leva em consideração alguns critérios, como idade, tempo de menopausa ou hipogonadismo, sintomas, dores, vias de administração, comorbidades, dentre outros. Portanto, os médicos passaram a avaliar a TRH de forma individualizada, e atualmente, essa conduta continua sendo aplicada, onde primeiro são avaliados os aspectos individuais do paciente e, em seguida, é escolhida a terapia de acordo com a relação risco/benefício (Lima et al., 2020). Atualmente existem várias formulações disponíveis para a veiculação da TRH, como adesivos, gel transdérmico, injeções intramusculares, e formulações orais. Contudo, foi observado que a via transdérrmica é interessante para a TRH, pois permite aplicar e remover a formulação de forma fácil, evita o efeito de primeira passagem, reduz os efeitos adversos, via não invasiva, e aumenta adesão ao tratamento. Por isso, a via transdérmica na veiculação de hormônios tem sido cada vez mais explorada (Singh et al., 2015).

Dentre as formulações utilizadas para a TRH, pela via transdermica, destaca-se os géis, que são sistemas semissólidos constituídos por uma dispersão de pequenas partículas inorgânicas ou macromoléculas orgânicas disperso em um veículo líquido (Allen et al., 2007). Esses sistemas são dispersões coloidais de aparência transparente e translúcidos, ou turvos, dependendo do grau de dispersão molecular, inodoros, possuem aspecto seco, não oclusivos, e raramente causam irritação à pele (Carniél, 2013). No caso da utilização de géis para a TRH, estes podem estar disponíveis como sachês de dose única préembalados ou sistemas multidoses, ou também podem estar associados a uma matriz adesiva produzindo assim, um sistema de liberação modificada (Cunha, 2020). O gel de testosterona transdérmica mais comercializado é o Androgel TM, que é uma formulação de gel hidroalcoólico contendo 1\% p/p de testosterona (Hadgraft \& Lane, 2015; Johansen et al., 2020). Porém, a liberação de hormônios pela via transdérmica utilizando géis pode apresentar como desvantagens: alta variabilidade interindividual na absorção do fármaco, devido à diferença de absorção e dos locais de aplicação. Apesar disso, os géis dominam o mercado da TRH, possivelmente por causa da sua maior aceitabilidade pelo paciente, natureza não oclusiva, facilidade de aplicação, remoção, manutenção dos níveis plasmáticos, e a possibilidade de estarem associados a uma matriz modificando o perfil de liberação do fármaco (Gomes \& Boldo, 2020; Kamal et al., 2020).

Dessa forma, o objetivo desse trabalho foi realizar uma revisão da literatura acerca da segurança e a eficácia dos géis transdérmicos na veiculação de três hormônios esteroides sexuais (estrogênio, progesterona e testosterona) utilizados na terapia de reposição hormonal.

\section{Metodologia}

Este estudo foi realizado por meio de uma revisão sistemática da literatura utilizando as seguintes plataformas: Google Acadêmico, Pubmed e Periódicos Capes, e foram selecionados os artigos dos últimos 10 anos. Inicialmente foram pesquisadas as formas e vias de administração dos hormônios esteroides utilizados na terapia de reposição hormonal, bem como as vantagens de sua utilização, e foi introduzido nesse trabalho o gel como veículo para a via transdérmica. Para a busca na literatura, foram utilizadas as seguintes palavras-chave em inglês e português relacionadas ao tema: via transdérmica, géis na veiculação de hormônios, Géis transdérmicos, Segurança e eficácia de géis transdérmicos, Hormônios veiculados pela via transdérmica, Reposição hormonal, Gel de Estrogênio, Transdermal route of drug administration, Transdermal patch, Safety and efficacy of transdermal gels, hormones carried by the transdermal route, hormone replacement transdermal gel, transdermal hormones, Testosterone gel, Estrogen hormone replacement, Progesterone effects.

Os critérios de inclusão e exclusão utilizados para a escolha dos trabalhos foram associados aos artigos mais recentes, 
publicados nos últimos 10 anos, com prioridade aos estudos mais referenciados com dados consubstanciados a parâmetros farmacocinéticos e clínicos que avaliaram a eficácia e segurança da formulação de géis transdérmicos na veiculação de hormônios esteroides sexuais em indivíduos com concentrações suprafisiológicas dos hormônios sexuais endógenos.

\section{Resultados e Discussão}

A Figura 1 mostra os principais resultados obtidos na revisão sistemática da literatura.

Figura 1. Fluxograma da triagem da revisão sistemática.

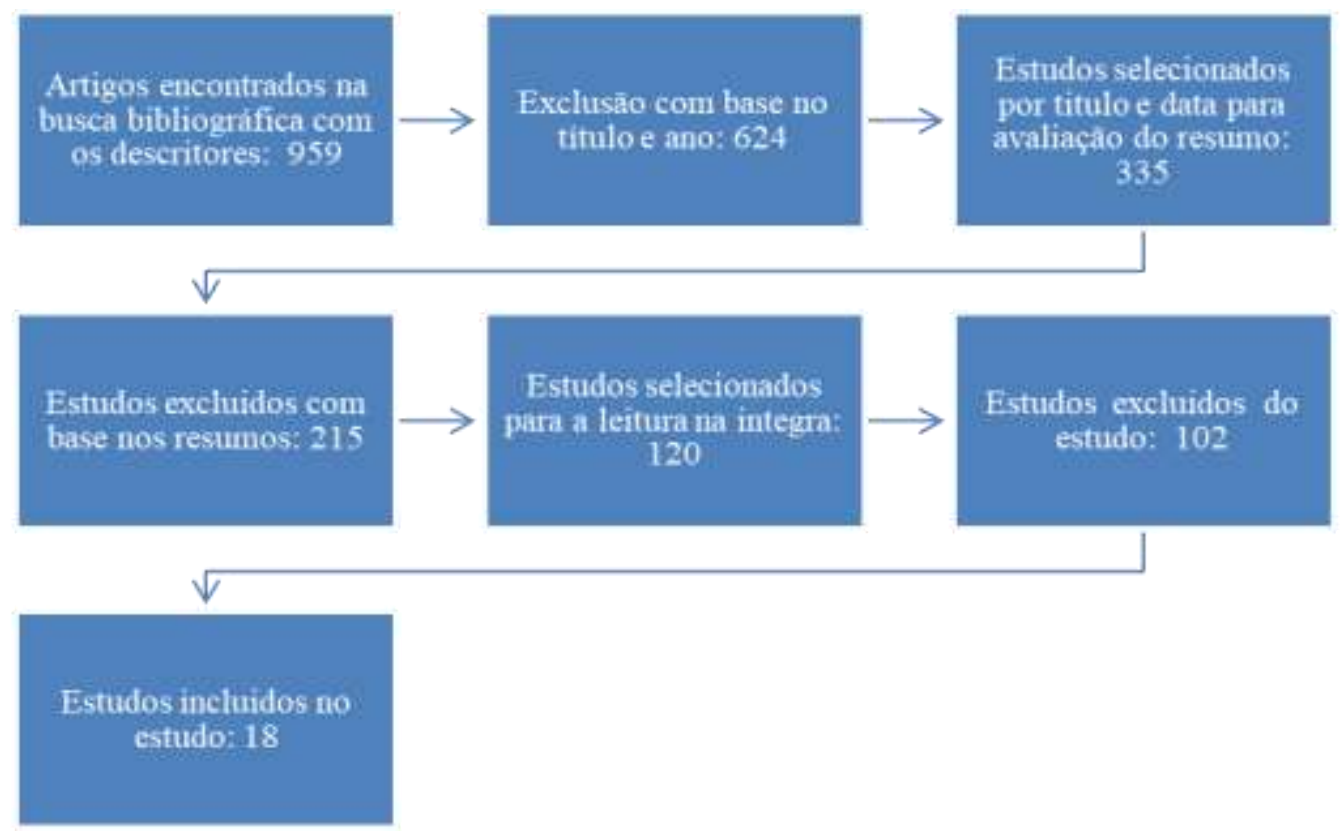

Fonte: Autores.

Foram identificados 959 estudos com potencial para serem incluídos no trabalho. Após a realização a avaliação do titulo e do ano de publicação, 335 artigos foram selecionados para a leitura do resumo, e 624 excluídos, pois os estudos estavam fora do corte temporal (2011-2021), e não respondiam a questão norteadora do trabalho. Para a leitura do resumo foram selecionados 335 artigos, e após a leitura do resumo foram excluídos 215 artigos, pois os trabalhos não avaliavam a eficácia e segurança dos géis transdérmicos, os parâmetros de farmacocinética, a absorção do gel no local de aplicação, sua segurança, o risco de transferência interpessoal de gel e níveis séricos suprafisiológicos hormonais. Foram selecionados 120 artigos para a leitura na integra, e após analise foram excluídos 102 artigos e incluídos 18, que responderam as questões norteadoras do trabalho.

Sendo assim, os estudos abordados investigam a proporção do número de pacientes respondentes a TRH, que obtiveram seus níveis séricos hormonais normalizados, e também a manifestação dos sintomas clínicos e a presença de efeitos adversos, ponderando os benefícios em relação ao uso da TRH a fim de se obter a satisfação do paciente com o tratamento, bem como a eficácia e adesão ao tratamento.

\section{Testosterona}

O Quadro 1 apresenta os estudos que avaliaram a eficácia e a segurança de géis transdérmicos de testosterona. Todos os trabalhos foram realizados em indivíduos hipogonadais, portanto, os parâmetros para a eficácia foram definidos em termos da porcentagem de indivíduos alcançando a concentração sérica de testosterona dentro da faixa normal de 300-1.000 ng/dL, 
por meio de análises farmacocinéticas que avaliaram os níveis de testosterona total, livre e biodisponível. A segurança foi avaliada pela capacidade das formulações manterem os níveis séricos de testosterona, com a redução dos picos de concentrações máximas de testosterona sérica (Cmáx), por meio da avaliação dos parâmetros qualitativos, como Índice Internacional de Função Erétil (IIEF), questionário de avaliação multidimensional de fadiga (MAF), exames clínicos (ECGs, EDR, PSA, testes de função hepática, lipídios e hematologia), e pelo aparecimento de eritemas no local de aplicação e eventos adversos relacionados.

Quadro 1: Artigos que avaliaram à eficácia e a segurança do uso de gel de transdérmico de testosterona.

\begin{tabular}{|c|c|c|c|c|c|}
\hline \multirow[b]{2}{*}{ Parâmetros } & \multicolumn{5}{|l|}{ Estudos } \\
\hline & Sansone (2019) & Kaufman (2011) & Dobs (2012) & Efros (2016) & Belkoff (2018) \\
\hline Amostragem & $\begin{array}{l}30 \text { indivíduos } \\
\text { hipogonadais }\end{array}$ & $\begin{array}{l}234 \text { indivíduos (40 } \\
\text { grupo controle) }\end{array}$ & $\begin{array}{l}138 \text { indivíduos } \\
\text { hipogonadais }\end{array}$ & $\begin{array}{c}40 \text { homens } \\
\text { hipogonadais ( } 20 \\
\text { aplicação manual e } \\
20 \text { uso de aplicador) }\end{array}$ & $\begin{array}{l}180 \text { indivíduos } \\
\text { hipogonadais }\end{array}$ \\
\hline $\begin{array}{l}\text { Tempo de } \\
\text { duração }\end{array}$ & Não especificado & 182 dias & 90 dias & Não especificado & 9 meses \\
\hline $\begin{array}{l}\text { Doses/ } \\
\text { aplicação }\end{array}$ & $\begin{array}{l}\text { Gel de testosterona } \\
2 \% \\
\text { Dose: } 40 \mathrm{mg} / \mathrm{dia} . \\
\text { Aplicação manual, } \\
\text { ombros ou } \\
\text { antebraços. }\end{array}$ & $\begin{array}{c}\text { Gel de testosterona } \\
1,62 \% \text {. Doses } \\
\text { tituladas }(1,25 \mathrm{~g}, 2,5 \\
\mathrm{g}, 3,75 \mathrm{~g} \text { ou } 5,0 \mathrm{~g},) \\
\text { Aplicação manual } \\
\text { na parte superior do } \\
\text { braço/ombros ou } \\
\text { abdômen. }\end{array}$ & $\begin{array}{c}\text { Gel de } \\
\text { testosterona } 2 \% \text {, } \\
\text { em doses tituladas } \\
\text { com incrementos } \\
\text { de } 10 \mathrm{mg} \text {, na faixa } \\
\text { de } 10 \mathrm{mg} \text { até } 70 \\
\mathrm{mg} .\end{array}$ & $\begin{array}{c}\text { Gel de } \\
\text { testosterona Doses: } \\
\text { 1,25, 2,50 e } 3,75 \mathrm{ml} \\
(23,46 \text { e } 70 \mathrm{mg}) \\
\text { Aplicação } \\
\text { manual/aplicador: } \\
\text { coxa, abdômen e } \\
\text { ombro/braço. }\end{array}$ & $\begin{array}{l}\text { Gel de testosterona } \\
2 \% . \\
\text { Doses tituladas: } \\
\text { mínima } 23 \mathrm{mg} \text { e } \\
\text { máxima } 69 \mathrm{mg} . \\
\text { Aplicação: uso de } \\
\text { aplicador. }\end{array}$ \\
\hline Objetivo & $\begin{array}{l}\text { Monitorar a } \\
\text { dosagem/ } \\
\text { manutenção dos } \\
\text { níveis séricos de } \\
\text { testosterona na faixa } \\
\text { fisiológica normal, } \\
\text { em pacientes } \\
\text { hipogonadais. }\end{array}$ & $\begin{array}{l}\text { Avaliar a eficácia e } \\
\text { segurança de doses } \\
\text { tituladas de gel de } \\
\text { testosterona em } \\
\text { homens } \\
\text { hipogonadais }\end{array}$ & $\begin{array}{l}\text { Restaurar as } \\
\text { concentrações } \\
\text { séricas de } \\
\text { testosterona aos } \\
\text { níveis fisiológicos } \\
\text { e minimizar os } \\
\text { sinais e sintomas } \\
\text { hipogonadais } \\
\text { masculino. }\end{array}$ & $\begin{array}{c}\text { Avaliar a } \\
\text { farmacocinética, } \\
\text { farmacodinâmica e } \\
\text { segurança do gel de } \\
\text { testosterona, em } \\
\text { homens } \\
\text { hipogonadais, } \\
\text { usando dois } \\
\text { métodos de } \\
\text { aplicação. }\end{array}$ & $\begin{array}{l}\text { Avaliar a eficácia, } \\
\text { segurança, } \\
\text { tolerabilidade local e } \\
\text { farmacocinética do gel } \\
\text { de testosterona em } \\
\text { homens hipogonadais. }\end{array}$ \\
\hline Eficácia & $\begin{array}{c}36,7 \% \text { dos } \\
\text { indivíduos } \\
\text { permaneceram com } \\
\text { os níveis séricos de } \\
\text { testosterona dentro } \\
\text { da faixa normal após } \\
23 \mathrm{~h} .\end{array}$ & $\begin{array}{c}75 \% \text { dos } 234 \\
\text { indivíduos } \\
\text { hipogonadais } \\
\text { tratados atingiram os } \\
\text { níveis normais de } \\
\text { testosterona ao } \\
\text { longo do estudo. }\end{array}$ & $\begin{array}{c}\text { O gel } \\
\text { de testosterona } \\
\text { aplicado uma vez } \\
\text { ao dia, restaurou } \\
\text { os níveis de } \\
\text { testosterona em } \\
\text { mais de } 75 \% \text { dos } \\
\text { pacientes. }\end{array}$ & $\begin{array}{l}\text { Após múltiplas } \\
\text { aplicações manuais } \\
\text { cerca de } 70 \% \text { dos } \\
\text { indivíduos } \\
\text { responderam nas } 3 \\
\text { doses. } \\
\text { Melhora nos } \\
\text { domínios de MAF e } \\
\text { IIFE, exceto para a } \\
\text { função erétil. }\end{array}$ & $\begin{array}{l}52,8 \% \text { dos indivíduos } \\
\text { responderam } \\
\text { no primeiro dia de } \\
\text { tratamento. Com a } \\
\text { continuação do } \\
\text { tratamento a resposta } \\
\text { atingiu } 82,1 \%\end{array}$ \\
\hline Segurança & $\begin{array}{c}\text { Não foram } \\
\text { observados efeitos } \\
\text { adversos e não } \\
\text { houve pico de } \\
\text { testosterona sérico. }\end{array}$ & $\begin{array}{c}10 \text { indivíduos } \\
\text { apresentaram C máx } \\
\text { de testosterona } \\
\text { sérica maior que } \\
2.500 \mathrm{ng} / \mathrm{dL} . \\
55,6 \% \text { dos } \\
\text { indivíduos } \\
\text { apresentaram pelo } \\
\text { menos um evento } \\
\text { adverso grave } \\
\text { comparado com } \\
\text { 37,5\% para o grupo } \\
\text { placebo. }\end{array}$ & $\begin{array}{l}\text { Eventos adversos } \\
\text { comuns, como } \\
\text { reações cutâneas } \\
\text { leves e } \\
\text { moderadas. Não } \\
\text { houve eventos } \\
\text { adversos graves. }\end{array}$ & $\begin{array}{l}\text { Aparecimento de um } \\
\text { leve eritema na parte } \\
\text { interna da coxa. } \\
\text { Na aplicação de } \\
\text { múltiplas doses } \\
\text { houve eventos } \\
\text { adversos leves, e } \\
\text { aumento dos níveis } \\
\text { séricos do PSA. }\end{array}$ & $\begin{array}{c}\text { A pontuação total } \\
\text { média do IIEF e MAF } \\
\text { mostrou uma melhora } \\
\text { significativa. } 30,2 \% \\
\text { dos indivíduos } \\
\text { apresentaram Cmáx de } \\
\text { testosterona maior que } \\
1500 \text { ng/dl, e foram } \\
\text { submetidos ao ajuste } \\
\text { da dose. }\end{array}$ \\
\hline
\end{tabular}

Fonte: Autores.

Sansone (2019) avaliou os níveis séricos de testosterona em 30 indivíduos hipogonadais tratados com gel transdérmico de testosterona $2 \%$ (40 mg/dia), e comparou com indivíduos saudáveis. A eficácia do tratamento foi avaliada por meio de duas coletas sanguíneas, no tempo de 2 h e 23 h, após a aplicação manual do gel, e após cinco dias. A taxa de absorção de testosterona não mostrou flutuações, e foi praticamente constante em cada indivíduo, assim como também a de testosterona 
livre e biodisponível. Os resultados mostraram que $2 \mathrm{~h}$ após a aplicação do gel, 70\% dos indivíduos (21 pacientes) atingiram testosterona sérica total média maior que 3,5 ng/ml, e após $23 \mathrm{~h}$ apenas 36,7\% dos indivíduos (11 pacientes) ainda apresentavam testosterona total sérica adequada, indicando que uma minoria dos indivíduos mantém testosterona sérica adequada após $23 \mathrm{~h}$ (Sansone et al., 2019). As medições no tempo de $2 \mathrm{~h}$ e $23 \mathrm{~h}$ apresentaram níveis séricos significativamente mais baixos, quando comparados entre si, para a testosterona total, livre e biodisponível, e resultaram na absorção de testosterona pelo organismo. Dessa forma, como níveis séricos de testosterona adequados para a TRH não foram mantidos a continuação do tratamento não é recomendada.

Contudo, esse estudo não considerou as características demográficas, como raça, idade, índice de massa corporal (IMC), antígeno prostático específico, sendo assim, parâmetros importantes que estão envolvidos na absorção de testosterona, como IMC, deve ser considerado, uma vez que eles podem impactar a eficácia e segurança das formulações (Kaufman et al., 2011). Também não foram realizados exames clínicos necessários para os estudos de segurança, e apenas foram realizados os exames do hematócrito e antígeno prostático específico (PSA). A dose utilizada nesse estudo foi a dose mínima recomendada de testosterona, $40 \mathrm{mg} / \mathrm{dia}$, e talvez um aumento da dose, utilizando um esquema de doses tituladas poderia alcançar resultados mais adequados para atingir o nível de testosterona sérica desejável.

Efros (2016) realizou um estudo utilizando doses múltiplas de 1,25, 2,50 e 3,75 ml, que equivalem a 23, 46 e $70 \mathrm{mg}$ de testosterona, respectivamente, de um gel de testosterona à $2 \%$. Esse estudo obteve melhores resultados quanto à taxa de resposta dos indivíduos submetidos a essa terapia. Nesse estudo, as doses foram aplicadas com a mão e com auxílio de um aplicador, em três locais diferentes: braço/ombro, coxa e abdômen, em 40 homens hipogonadais, distribuídos em grupos de 20 para cada tipo de aplicação. O grupo que utilizou a aplicação manual mostrou uma maior absorção de testosterona na região superior do braço/ombro, comparado com as regiões da coxa e abdômen, e foi acompanhado por um aumento proporcional da concentração média de testosterona sérica. Os resultados mostraram que após múltiplas aplicações manuais cerca de $70 \%$ dos indivíduos responderam nas três doses administradas. A avaliação farmacocinética investigou a faixa fisiológica normal dos níveis de testosterona sérica (Cmin); a concentração média do estado estacionário de testosterona sérica, como também o perfil de segurança dessa nova formulação comparando os diferentes métodos de aplicação. Observou-se que quatro indivíduos apresentaram uma concentração média de testosterona sérica acima do estado estacionário, e seis participantes apresentaram a concentração máxima de testosterona elevada, porém nenhum indivíduo obteve a concentração abaixo dos níveis considerados normais (Efros et al., 2016).

A eficácia e segurança também foram avaliadas pelo IIEF e MAF, parâmetros que atestam a segurança da terapia e indicam a melhora da qualidade de vida do paciente. Além disso, para avaliar a segurança também foram considerados os efeitos adversos no local de aplicação e a ocorrência de irritabilidade, alterações nos exames clínicos, tais como, sinais vitais, exame físico e níveis séricos de PSA. O grupo que realizou a aplicação manual do gel apresentou melhora significativa nos escores médios para todos os domínios relevantes da função sexual masculina, exceto para a função erétil, de acordo com o questionário de funcionamento sexual IIEF. Também houve melhora significativa na fadiga para todos os domínios do MAF. Quanto à segurança, após a aplicação de uma dose única manual de $2,5 \mathrm{~mL}$ de gel de testosterona houve apenas um evento adverso com o aparecimento de um leve eritema, na parte interna da coxa, 24 h após a aplicação. Em contrapartida, após a aplicação das múltiplas doses ocorreram eventos adversos leves tais como, aumento da alanina transaminase, desconforto gastrointestinal, dor no mamilo, e aumento dos níveis séricos do PSA (Efros et al., 2016).

Sendo assim, foram observados resultados positivos em relação à eficácia quando foram utilizadas doses tituladas do gel de testosterona para o tratamento em homens hipogonadais. Esse efeito foi pronunciado no grupo que utilizou o aplicador para o gel, pois permitiu a administração de uma dosagem precisa e apresentou melhor adesão e aceitação. As avaliações sobre a segurança, por meio do IIFE e MAF, indicaram uma melhora na qualidade de vida do paciente com a redução dos sintomas 
decorrentes dos baixos níveis de testosterona sérica. Porém, a segurança dos níveis de testosterona suprafisiológicos deve melhorar, uma vez que no estudo com a aplicação manual quatro indivíduos apresentaram concentração média de testosterona sérica acima do estado estacionário, e no estudo com o uso do aplicador seis participantes apresentaram a concentração máxima de testosterona acima dos níveis normais. Isso indica que esses resultados suprafisiológicos dos níveis de testosterona séricos devem ser monitorados em longo prazo, sendo assim através da titulação das doses deve ser observado os níveis estacionários da concentração de testosterona sérica. Apesar disso, os eventos adversos encontrados foram leves e a maioria deles está relacionada com a terapia (Efros et al., 2016).

Kaufman (2011) desenvolveu um gel de testosterona $(1,62 \%)$ com uma viscosidade alta, um menor volume de aplicação, maior permeação, e com níveis de testosterona séricos seguros e eficazes, para homens hipogonadais, e avaliou o seu desempenho usando doses tituladas durante 182 dias. O autor mostrou resultados mais consistentes com uma resposta dentro da faixa normal diária de testosterona ao longo do estudo, em 75\% dos indivíduos (Kaufman et al., 2011). Para avaliar a eficácia foram realizados estudos farmacocinéticos, onde foram medidas todas as formas de testosterona presentes no soro, incluindo espécies livres e reversivelmente ligadas a proteínas (globulina de ligação ao hormônio sexual [SHBG] e albumina ligada). Outras medidas secundárias incluíram medidas de hormônio luteinizante (LH), hormônio folículo-estimulante (FSH) e marcadores séricos de risco inflamatório e cardiovasculares selecionados (fator de necrose tumoral- $\alpha$, interleucina-6, interleucina-10 [IL-10], proteína $\mathrm{C}$ reativa de alta sensibilidade), e também marcadores séricos de metabolismo ósseo (fosfatase alcalina específica do osso e c-telopeptídeo reticulado tipo 1).

Os parâmetros farmacocinéticos mostraram a concentração média de estradiol dentro do limite normal nos dias 14, 56, 112 e 182 para todos os grupos de tratamento, exceto para o placebo e grupos de 1,25 g, e no dia 56, o qual estava ligeiramente acima do limite superior da faixa normal. Esse resultado corrobora o sucesso da terapia, uma vez que, o estradiol nos homens é essencial para modular a libido, a função erétil e a espermatogênese, e níveis baixos de testosterona e de estrogênio elevado aumentam a incidência de disfunção erétil independentemente um do outro (Schulster et al., 2016). Os níveis de SHBG, LH e FSH diminuíram significativamente após o uso do gel de testosterona. A globulina de ligação ao hormônio sexual (SHBG) foi diminuída pelo aumento dos níveis de testosterona sérica disponíveis, visto que a maior parte da testosterona em circulação fica ligada à globulina de ligação ao hormônio sexual (SHBG). Esta globulina é responsável pelo equilíbrio da testosterona circulante, pois a testosterona possui a característica de manter uma ligação fraca com a albumina do soro e dissocia-se livremente na circulação sanguínea sendo disponível para a absorção pelos tecidos. A fosfatase alcalina sérica específica do osso, um marcador bioquímico da formação óssea, aumentou significativamente, porém esse aumento não foi significativo, quando comparado ao placebo. O telopeptídeo $\mathrm{C}$ reticulado tipo 1 sérico, um marcador bioquímico de reabsorção óssea, diminuiu significativamente quando comparado ao grupo de placebo (Kaufman et al., 2011).

Esses resultados indicam uma boa resposta ao tratamento, com o efeito preventivo da manutenção da densidade mineral óssea promovida pelo uso da testosterona. Uma vez que os andrógenos, direta ou indiretamente através dos estrógenos, preservam o osso trabecular principalmente pela diminuição da osteoclastogênese, e ambos os hormônios neutralizam a apoptose dos osteoblastos e estimulam a apoptose dos osteoclastos, sugerindo que os andrógenos e estrógenos circulantes são protetores dos ossos (Mohamad, 2016).

A segurança clínica foi avaliada registrando os eventos adversos e sinais vitais tais como, eletrocardiogramas (ECGs), exame retal digital (EDR) e escore internacional de sintomas prostáticos (IPSS-1), PSA, além da coleta periódica de avaliações clínicas laboratoriais. Os eventos adversos graves emergentes da terapia são: dor nas costas, enfarte do miocárdio, taquicardia, diarreia, dispepsia, gastroenterite, pneumonia, queda, diabetes mellitus, tumor pituitário, dor radicular, aumento da libido, distúrbio do sono e aumento da ereção. O percentual de indivíduos do grupo que utilizou gel de testosterona e apresentou reações adversas graves $(2,1 \%)$ foi semelhante ao do grupo placebo (2,5\%). Além disso, 55,6\% dos indivíduos apresentaram 
pelo menos um evento adverso grave, com 10 indivíduos atingindo a concentração máxima de testosterona sérica maior que $2.500 \mathrm{ng} / \mathrm{dL}$. Dentre os resultados para a avaliação da segurança, além dos dados farmacocinéticos, os resultados dos testes e avaliações clínicas não demonstraram alterações significativas (Kaufman et al., 2011). Portanto, os indivíduos alcançaram os níveis séricos normais de testosterona, que resultou no alívio dos sintomas hipogonadais, com a melhora da função sexual, redução da massa corporal e aumento da densidade mineral óssea.

Esse estudo apresentou uma amostragem maior, com critérios de seleção mais rigorosos, o que permitiram equiparar as diferenças interindividuais, como o IMS, raça, idade e sexo, que são frequentes variáveis nos estudos. Os indivíduos hipogonadais submetidos ao tratamento não apresentaram irritação nos locais de aplicação do gel. As doses tituladas utilizadas também permitiram uma melhor escolha da dose final do tratamento, indicando que o tratamento em doses tituladas pode ser um bom caminho para se garantir a segurança da terapia. Porém, apesar da eficácia e de altas taxas de adesão do paciente, a segurança continua sendo um fator crítico, uma vez que a terapia mostrou alcançar os níveis médios séricos de testosterona adequados, porém muitos indivíduos apresentaram picos na Cmáx de testosterona sérica, que resultaram em efeitos adversos indesejáveis, além dos riscos associados ao desenvolvimento de câncer de próstata e distúrbios cardiovasculares.

Belkoff (2018) realizou um estudo utilizando gel de testosterona a $2 \%$ com auxílio de um aplicador, com doses tituladas, e acompanhou 180 indivíduos hipogonadais por 9 meses. Nesse estudo, a eficácia do gel de testosterona foi bem estabelecida com a continuação do tratamento. Observou-se que no primeiro dia de tratamento 52,8\% dos indivíduos responderam a terapia, e com a continuação do tratamento a resposta atingiu 82,1\% dos indivíduos. Belkoff (2018) obteve resultados em dois estudos multicêntricos de fase 3, o primeiro com duração de 3 meses e o segundo com a duração de 6 meses, totalizando 9 meses de estudo. O seu trabalho posterior (6 meses) avaliou a segurança do gel de testosterona em pacientes que passaram pelo primeiro estudo ( 3 meses). Nesse trabalho também foi observado que a $\mathrm{C}$ máx de testosterona foi maior que $1500 \mathrm{ng} / \mathrm{dl}$, em 31,8\% dos indivíduos na primeira parte do estudo, e em 12,7\% dos indivíduos na segunda parte do estudo. A pontuação total média do IIEF e MAF mostrou uma melhora significativa nos sintomas ocasionados pelos baixos níveis de testosterona séricos (Belkoff et al., 2018).

No estudo de Belkoff (2018), a dose inicial do gel de testosterona foi de $46 \mathrm{mg}$, aplicada na parte superior do braço/ombro, e para a primeira parte do estudo o ponto final foi a taxa de resposta de indivíduos com concentração média de testosterona sérica 24 h de 300-1050 ng/dL, após 90 dias. Sendo assim, 127 pacientes concluíram o estudo com a taxa de resposta a terapia de 85,5\%, e esse resultado foi atribuída à formulação hidroalcoólica, de rápida absorção na pele, e a utilização de um aplicador que facilitou a administração e reduziu os riscos da exposição secundária à testosterona. $\mathrm{O}$ percentual de indivíduos que alcançaram a C máx maior que $1500 \mathrm{ng} / \mathrm{dL}$ pode ser explicado pela titulação pré-dose de testosterona, a qual pode ter resultado em uma titulação de uma dose mais alta do que o necessário, em alguns indivíduos. Porém, a decisão de titulação foi ajustada posteriormente baseando-se nos níveis de testosterona sérica pós-dose de 4 horas, consistentes com o tempo até a concentração máxima de testosterona, ao invés de níveis de testosterona sérica pré-dose, o que pode ter reduzido o número de indivíduos com níveis suprafisiológicos de testosterona, 31,8\%, na primeira parte do estudo, para $12,7 \%$ ao final do tratamento. Com relação à segurança, os indivíduos não obtiveram aumentos notáveis no antígeno específico da próstata (PSA) e apresentaram poucas reações na pele. A pontuação total média do IIEF e MAF mostrou uma melhora significativa nos sintomas ocasionados pelos baixos níveis de testosterona séricos, resultando na melhora da qualidade de vida dos indivíduos, indicando o sucesso da terapia (Belkoff et al., 2018).

Dessa forma, o estudo realizado por Belkoff (2018) foi o que apresentou os melhores resultados quanto à eficácia e a segurança do tratamento. Nesse sentido, a concentração máxima de testosterona sérica e a avaliação da ocorrência dos eventos adversos é o parâmetro principal para a garantia da segurança do uso de géis transdérmicos. Todavia, nenhum trabalho descrito nesse estudo foi capaz de evitar níveis séricos máximos de testosterona, apesar de terem obtido reduções conforme mostra o 
estudo de Belkoff (2018). No entanto, existem preocupações com relação aos efeitos adversos de doses prolongadas e suprafisiológicas de testosterona, uma vez que as diretrizes recomendam evitar níveis anormalmente elevados sustentados, o qual deve se manter dentro da faixa fisiológica considerada normal entre 300 a $1000 \mathrm{ng} / \mathrm{dL}$. Recomenda-se que durante a TRH, a testosterona seja dosada para garantir a manutenção da sua concentração sérica dentro da faixa normal (Dobs et al., 2012). Por isso, a maioria dos estudos analisados realizou um esquema de titulação de doses obtendo resultados mais seguros.

Dobs (2012) investigou a eficácia, o perfil farmacocinético e a segurança de um gel de testosterona $2 \%$, denominado Fortesta gel (Tostran, Tostrex e Itnogen), aplicado na parte frontal e interna das coxas, com uma dose inicial de $40 \mathrm{mg}$ de testosterona e ajustada em incrementos de $10 \mathrm{mg}$ para flexibilidade da dose, com uma dose mínima de $10 \mathrm{mg}$ e uma dose máxima de $70 \mathrm{mg}$ de testosterona. O estudo mostrou que $75 \%$ dos pacientes alcançaram níveis de testosterona dentro da faixa fisiológica normal, após 24 h. 94,6\% dos pacientes apresentaram níveis de C máx de até $1500 \mathrm{ng} / \mathrm{dL}$, porém 1,6\% pacientes ainda apresentaram valores entre 1800 e $2500 \mathrm{ng} / \mathrm{dL}$, indicando que apesar da redução dos picos de Cmáx de testosterona estes não puderam ser evitados. Esse estudo mostrou que o esquema de doses dosimetradas foi vantajoso, pois permitiu o ajuste da dose, em pequenos incrementos, forneceu a dosagem individualizada para os pacientes, alcançando as concentrações eugonadais, e foi capaz de evitar as concentrações suprafisiológicas de testosterona. Sendo assim, apresentou um melhor perfil de eficácia.

Com relação aos parâmetros secundários para a avalição da segurança (IIEF e MAF), clínicos (ECGs, EDR, PSA, testes de função hepática, lipídios e hematologia), aparecimento de eritemas no local de aplicação e eventos adversos relacionados, todos os estudos não apresentaram alterações significativas que indicassem risco do paciente submetido à terapia (Dobs et al., 2012).

Os dados representados nos estudos indicam que a TRH com a testosterona é eficaz, porém ainda são necessários estudos em longo prazo que acompanhem as consequências dessa terapia, mantendo o ajuste de doses e exames periódicos para a garantia da segurança e a efetividade do tratamento. O acompanhamento deve ser individual ao paciente, e após o diagnóstico, o tratamento é prescrito com base na análise dos resultados dos exames junto com os sinais e sintomas clínicos.

Os estudos mostraram que as formulações transdérmicas de gel de testosterona apresentam maior eficácia quando há o ajuste das doses. A titulação das doses e as diferenças nas formulações vêm alcançando resultados promissores quanto à manutenção dos níveis séricos fisiológicos de testosterona. Os picos de Cmáx de testosterona continuam sendo um fator crítico relacionado à terapia. Portanto, a segurança precisa ser avaliada devido aos riscos associados a eventos cardiovasculares, e incidência de câncer de próstata relacionado aos níveis suprafisiológicos de testosterona sérica (Farwell et al., 2010).

\section{Estrogênio}

Para a terapia de reposição hormonal (TRH), o estrogênio ou a progesterona têm sido administrados em formulações orais, injetáveis ou transdérmicas. Porém, o uso oral exclusivamente de estrogênio derivado de equinos conjugados (EEC) é um fator crítico para a segurança no tratamento de sintomas vasomotores em mulheres com risco basal de doença cardiovascular (DCV) e câncer de mama (L'Hermite, 2017). Contudo, dentre os estudos que indicam perfis de associação estrogênio/progesterona se destacam o uso de hormônios bioidênticos, que são naturais endógenos que aliado a TRH via transdérmica parecem oferecer maior segurança ao paciente. Portanto, diante dos riscos associados à terapia estrogênica via oral, a via transdérmica tem sido uma alternativa promissora, e sua eficácia é descrita por ser capaz de diminuir os sintomas indesejáveis da menopausa, como também ser capaz de manter esses efeitos em longo prazo sem trazer riscos ao paciente (Archer et al., 2012)

O Quadro 2 mostra os artigos selecionados que avaliou a eficácia de géis transdérmicos de estrogênio no tratamento de mulheres menopausais, considerando os níveis séricos hormonais, o alívio dos sintomas vasomotores, enfrentamentos, 
humor depressivo, irritabilidade, exaustão física e mental, problemas sexuais, dentre outros aspectos relacionados ao climatério. A avaliação da segurança foi relacionada aos riscos associados a eventos tromboembólicos venosos e ao impacto estrogênico na DMO e o risco de fraturas.

Quadro 2: Artigos que avaliaram à eficácia e a segurança do gel transdérmico de estrogênio.

\begin{tabular}{|c|c|c|c|c|}
\hline \multirow[b]{2}{*}{ Parâmetros } & \multicolumn{4}{|c|}{ Estudos } \\
\hline & Mizunuma (2011) & Archer (2012) & Kim (2014) & Kim (2019) \\
\hline Amostragem & $\begin{array}{l}177 \text { mulheres } \\
\text { menopausais. }\end{array}$ & $\begin{array}{l}\text { Estudo de fase } 3 \text { e } 4: 196 \\
\text { e } 277 \text { mulheres } \\
\text { menopausais. }\end{array}$ & $\begin{array}{l}149 \text { mulheres } \\
\text { menopausais. }\end{array}$ & $\begin{array}{l}26 \text { mulheres menopausais } \\
\text { para a tibolona, e } 31 \text { para } \\
\text { o gel de estrogênio. }\end{array}$ \\
\hline Tempo de duração & 24 semanas. & $\begin{array}{l}12 \text { semanas para cada } \\
\text { estudo. }\end{array}$ & 2 anos. & 6 meses. \\
\hline Doses/aplicação & $\begin{array}{l}\text { Dose padrão }(1,8 \mathrm{~g} / \mathrm{dia} \text {, } \\
\text { contendo E2 } 1,08 \mathrm{mg} / \mathrm{dia}) \\
\text { e baixa dose }(0,9 \mathrm{~g} / \mathrm{dia} \text {, } \\
\text { contendo E2 } 0,54 \mathrm{mg} / \mathrm{dia})\end{array}$ & $\begin{array}{l}\text { Fase 3: } 1,5 \mathrm{mg} \text { ou } 0,75 \\
\text { mg de estradiol } \\
\text { (EstroGel } 0,06 \%) \text { ou } \\
\text { placebo; Fase } 4: 0,375 \\
\text { mg ou } 0,27 \mathrm{mg} \mathrm{de} \\
\text { estradiol }(0,03 \% \text { gel) ou } \\
\text { placebo. }\end{array}$ & $\begin{array}{l}\text { EEC via oral: } 0,625 \mathrm{mg} \text {; } \\
\text { estrogênio transdérmico: } \\
\text { adesivo de estradiol } 1,5 \\
\mathrm{mg} \text {; gel de estradiol } 1,5 \\
\mathrm{mg}(0,1 \%)\end{array}$ & $\begin{array}{l}2,5 \mathrm{mg} \text { de estrogênio - } \\
\text { tibolona via oral. } 1,5 \mathrm{mg} \\
\text { de estrogênio -gel } \\
\text { transdérmico associado } \\
\text { ao progestogênio ( } 10-20 \\
\text { mg de didrogesterona). }\end{array}$ \\
\hline Objetivo & $\begin{array}{l}\text { Avaliar a eficácia e a } \\
\text { segurança da terapia com } \\
\text { baixa dose de gel } \\
\text { transdérmico de estradiol } \\
\text { em mulheres japonesas } \\
\text { menopausais. }\end{array}$ & $\begin{array}{l}\text { Determinar a eficácia, } \\
\text { segurança, e a menor } \\
\text { dose utilizada no uso de } \\
\text { gel transdérmico de } \\
\text { estradiol para o } \\
\text { tratamento de mulheres } \\
\text { pós-menopáusicas } \\
\text { sintomáticas. }\end{array}$ & $\begin{array}{l}\text { Avaliar os efeitos da } \\
\text { terapia de estrogênio } \\
\text { transdérmico na DMO em } \\
\text { mulheres coreanas pós- } \\
\text { menopausa. }\end{array}$ & $\begin{array}{l}\text { Avaliar a eficácia da } \\
\text { tibolona e do estrogênio } \\
\text { transdérmico no } \\
\text { tratamento dos sintomas } \\
\text { em mulheres pós- } \\
\text { menopáusicas, com útero } \\
\text { intacto. }\end{array}$ \\
\hline Eficácia & $\begin{array}{l}\text { Mostrou eficácia na } \\
\text { inibição da recorrência de } \\
\text { afrontamentos. }\end{array}$ & $\begin{array}{l}\text { Mostrou eficácia em } \\
\text { todas as concentrações } \\
\text { testadas, porém a dose } \\
\text { de } 0,06 \%(0,75 \mathrm{mg} \text { de } \\
\text { estradiol) é a menor dose } \\
\text { prática para TRH. }\end{array}$ & $\begin{array}{l}\text { A terapia de estrogênio } \\
\text { transdérmico aumentou a } \\
\text { DMO, quando comparado } \\
\text { com o estrogênio via oral, } \\
\text { em mulheres coreanas } \\
\text { pós-menopáusicas. }\end{array}$ & $\begin{array}{l}\text { Ambas as opções } \\
\text { terapêuticas melhoraram } \\
\text { os sintomas. O estrogênio } \\
\text { transdérmico parece ser } \\
\text { mais eficaz na prevenção } \\
\text { do ganho de peso do que } \\
\text { a tibolona. }\end{array}$ \\
\hline Segurança & $\begin{array}{l}\text { Os eventos adversos no } \\
\text { grupo de baixa dose } \\
(21,6 \%) \text { foram } \\
\text { semelhantes à do placebo } \\
(22,5 \%) \text {, e menor do que } \\
\text { no tratamento de dose } \\
\text { padrão }(32,5 \%) \text {. }\end{array}$ & $\begin{array}{l}\text { Bem tolerado em ambos } \\
\text { os estudos, sendo a } \\
\text { maioria dos eventos } \\
\text { adversos leves ou } \\
\text { moderado. }\end{array}$ & $\begin{array}{l}\text { Após } 2 \text { anos, a terapia } \\
\text { aumentou } \\
\text { significativamente a } \\
\text { DMO na coluna lombar e } \\
\text { no quadril total, } \\
\text { comparado com os } \\
\text { controles. }\end{array}$ & $\begin{array}{l}\text { Não foram registrados } \\
\text { eventos adversos } \\
\text { relacionados à terapia } \\
\text { estrogênica. }\end{array}$ \\
\hline
\end{tabular}

Fonte: Autores.

Kim (2019) realizou um estudo comparativo entre a eficácia da tibolona (um medicamento oral utilizado na TRH) e do estrogênio transdérmico no tratamento dos sintomas da menopausa em mulheres pós-menopáusicas, com útero intacto. Nesse estudo 26 mulheres utilizaram tibolona, por via oral (2,5 mg de estrogênio), e 31 mulheres receberam gel de estrogênio transdérmico (1,5 mg de estrogênio) associado ao progestogênio (10-20 mg de didrogesterona). Os resultados foram avaliados pela escala de avaliação da menopausa para os sintomas menopausais na primeira consulta ambulatorial e após 6 meses. Após as análises entre os grupos, o estrogênio transdérmico foi capaz de reduzir os sintomas relacionados à menopausa equiparados à terapia por via oral, sendo encontrado o alívio para os sintomas como: humor depressivo, irritabilidade, exaustão física e mental, problemas sexuais e de bexiga e desconforto muscular e articular, enquanto o que desconforto cardíaco e secura vaginal melhoraram apenas no grupo de tibolona. Ambas as opções terapêuticas melhoraram os sintomas da menopausa, no entanto, o gel de estradiol produz níveis séricos de estradiol relativamente estáveis, o que foi observado comparativamente a níveis terapêuticos de estradiol presente em outras formulações, vias de administração e dosagens. Nesse estudo observou-se que a administração de estrogênio pela via transdérmica foi mais eficaz na prevenção do ganho de peso em mulheres na 
menopausa do que a tibolona (Kim et al., 2019). Sendo assim, Kim (2019) mostrou que o gel de estrogênio transdérmico foi eficaz com 1,5 mg de estrogênio associado a $10-20 \mathrm{mg}$ de progestogênio.

Archer (2012) realizou dois estudos de fase 3 e 4, com 196 mulheres que completaram o estudo de fase 3 com 1,5 mg ou 0,75 mg de estradiol (EstroGel 0,06\%) ou placebo, e 277 mulheres que completaram o estudo de fase 4 tratadas com 0,375 $\mathrm{mg}$ ou $0,27 \mathrm{mg}$ de estradiol ( $0,03 \%$ gel) ou placebo. Os dados mostraram a eficácia do gel de estradiol nas doses de $0,375,0,75$ e $1,5 \mathrm{mg}$ em ambos os estudos. Nesse trabalho foi considerada a frequência das ondas de calor típicas desse período climatérico, chamadas de fogachos ou afrontamentos, sendo de moderadas a graves, como também a citologia vaginal através das alterações do índice de maturação vaginal (IMV) (Archer et al., 2012). Os resultados dos estudos de fase 3 e 4 mostraram que o gel transdérmico de estradiol $0,06 \%$ (0,75 mg de estradiol) foi capaz de reduzir os sintomas menopausais, sendo esta considerada a menor dose prática para a terapia de reposição de estrogênio, no tratamento de sintomas vasomotores moderados a graves e atrofia vulvar e vaginal devido à menopausa. Foi observada a redução da frequência de afrontamentos moderados a graves, sendo a gravidade de todos os afrontamentos reduzida significativamente em relação ao placebo. Também houve a melhora do IMV, o qual foi expresso como a razão dos percentuais das células parabasais, intermediárias e superficiais, e permite a identificação da atrofia vaginal por meio do valor de maturação através do somatório e contagem dos esfregaços celulares, uma vez que o estrogênio é capaz aumentar a porcentagem de células superficiais e diminuir o predomínio de células parabasais decorrente da pós-menopausa. Portanto, melhoras significativas da linha de base no IMV foram observadas com estradiol na semana 12, além disso, os eventos adversos mais registrados foram cefaléia, infecção, dor mamária e náusea (estudo de fase 3) e insônia e cefaléia (estudo de fase 4), porém nenhum evento adverso grave foi relacionado ao tratamento. Sendo assim, o gel de estradiol se mostrou seguro, bem tolerado e eficaz na concentração de $0,06 \%$ (0,75 mg de estradiol) (Archer et al., 2012).

Mizunuma (2011) avaliou a eficácia e a segurança da terapia com gel transdérmico de estradiol para manutenção desse hormônio em mulheres japonesas menopausais. 177 mulheres que apresentavam um elevado número de ondas de calor diárias foram randomizadas para o tratamento com uma dose padrão de gel de estradiol transdérmico (E2) (1,8 g/dia, contendo E2 $1,08 \mathrm{mg} / \mathrm{dia})$, por 8 semanas, e metade da dose padrão ( $0,9 \mathrm{~g} / \mathrm{dia}$, contendo E2 $0,54 \mathrm{mg} / \mathrm{dia})$, ou placebo por 16 semanas. Os resultados mostraram uma melhora nas taxas do número de ondas de calor diárias, com um efeito inibidor sobre o surto de sintomas climatéricos e a redução da taxa de enfrentamentos diária. Observou-se que para o sucesso da terapia, com o alívio dos sintomas climatéricos, os pacientes precisam iniciar o tratamento com uma dose eficaz e, em seguida, mudar para uma dose mais baixa, após a melhora dos sintomas. Mizunuma (2011) testou essa hipótese e iniciou o tratamento com uma dose padrão de 1,08 mg/dia de E2, e após a melhora acentuada no número de ondas de calor, a dose de E2 foi reduzida pela metade $0,54 \mathrm{mg} / \mathrm{dia}$, a qual demonstrou ser eficaz e sustentar o efeito do estradiol em mulheres climatéricas. Além de reduzir a exposição de E2 em longo prazo, sendo ao mesmo tempo seguro com a diminuição da incidência de eventos adversos relacionados ao tratamento, quando comparado ao grupo com a dose padrão de E2. Dessa forma, a incidência de eventos adversos relacionados ao tratamento no grupo com baixa dose (21,6\%) foi semelhante à do placebo $(22,5 \%)$, mas foi menor do que no tratamento de dose padrão $(32,5 \%)$. A eficácia foi medida através de uma escala contendo a frequência do número médio de ondas de calor diárias, e comparadas com a linha de base de cada visita subsequente, denominadas como: melhora acentuada; melhora moderada; melhora leve; nenhuma mudança; ou piora. Portanto, a terapia de manutenção com baixa dose de estradiol aplicada a mulheres mostrou ser eficaz (inibição da recorrência), e seguro para o tratamento de distúrbios climatéricos e sintomas de deficiência de estrogênio (Mizunuma, 2011).

Renoux (2010) avaliou todos os casos de incidentes de tromboembolismo venoso (TEV) relacionados à terapia de reposição hormonal, entre janeiro de 1987 e março de 2008, no banco de dados de pesquisa de prática geral do Reino Unido. Os resultados mostraram que o risco de TEV não aumentou com o uso de estrogênio transdérmico sozinho ou combinado com 
um progestagênio. Por outro lado, o risco aumentou com o uso de estrogênio oral e estrogênio-progestogênio oral, acompanhado com o aumento da dosagem de estrogênio. Portanto, os riscos com formulações orais foram particularmente elevados durante o primeiro ano de uso, mas desapareceram 4 meses após a interrupção, enquanto que a terapia transdérmica não foi associada a um risco aumentado de TEV em mulheres na pós-menopausa (Renoux et al., 2010).

Sendo assim, qualquer via sistêmica não oral evita o efeito de primeira passagem diminuindo o impacto estrogênico no fígado, o qual estimula a cascata de coagulação (incluindo geração de trombina e resistência à proteína $\mathrm{C}$ ativada) e leva ao aumento do risco de eventos tromboembólicos venosos (L'Hermite, 2017). Esse efeito pode ser evitado pela administração desse hormônio pela pele, conforme demonstrado por Renoux (2010). Além disso, conforme mostrado nesse estudo, o estradiol transdérmico não confere nenhum risco adicional de eventos tromboembólicos venosos em mulheres com alto risco, como obesidade, mutações pró-trombótico e histórico pessoal desses eventos. Portanto, o estradiol transdérmico não é contraindicado nesses pacientes, que podem se beneficiar da terapia hormonal estrogênica pela via transdérmica, caso esta seja recomendada (Renoux et al., 2010).

Canonico (2016) avaliou o risco de derrame associado aos estrogênios orais e transdérmicos, e também a progesterona na TRH. Os resultados indicaram que os estrogênios transdérmicos podem ser a opção mais segura para o uso de terapia hormonal de curto prazo. Já que o risco de desfecho para os eventos isquêmicos se mostrou aumentado para o grupo de estrogênio via oral, bem como não houve associação desses eventos com o uso de progesterona (Canonico et al., 2016). L'Hermite (2017) reuniu os dados de dois estudos caso controle, os quais relataram risco aumentado de AVC isquêmico em usuárias de estrogênio oral, ao contrário do observado em usuárias de estradiol transdérmico, que não apresentaram esse risco. Os estudos indicaram que o uso oral exclusivamente de estrogênios derivados de equinos conjugados apresentou um efeito pronunciado, sendo um fator crítico para a segurança no tratamento de sintomas vasomotores em mulheres com risco basal de doença cardiovascular (DCV) e câncer de mama. Portanto, os resultados apontam que a via transdérmica de administração de estrogênio evita o excesso de eventos tromboembólicos venosos e acidente vascular cerebral isquêmico (Canonico, 2016; L'Hermite, 2017).

Os hormônios esteróides sexuais desempenham um papel fundamental no desenvolvimento e manutenção do esqueleto. Os níveis de estrogênios ovarianos estão associados à densidade mineral óssea (DMO), especialmente as frações livres ou biodisponíveis, que diminuem com o aumento da idade em homens e mulheres, e provavelmente contribuem para a perda óssea e fratura. Portanto, o estrogênio é o principal regulador do metabolismo ósseo em homens e mulheres e tem efeitos diretos sobre os osteócitos, osteoblastos e osteoclastos e efeitos inibitórios que bloqueiam a ativação dos osteoclastos diretamente ou via osteoblastos e células T. A ação final do estrogênio no esqueleto é diminuir a remodelação e reabsorção óssea, mantendo a formação óssea (Cauley, 2015)

Sendo assim, para avaliar o impacto do estradiol trandérmico na DMO, foram pesquisados estudos que avaliaram o risco de fratura, através de ensaios clínicos. Nesse contexto, a maioria dos trabalhos relata a manutenção e a melhora da densidade mineral óssea (DMO) relacionada ao uso de estradiol transdérmico. Kim (2014) investigou os efeitos da terapia de estrogênio transdérmico na densidade mineral óssea (DMO) em 149 mulheres coreanas na pós-menopausa. A reposição hormonal estrogênica por via transdérmica foi feita usando um adesivo, ou gel, e também estrogênio, por via oral, nas respectivas doses de: $1,5 \mathrm{mg}$ (adesivo), $1,5 \mathrm{mg}$ (0,1\% de gel de estradiol) e 0,625 $\mathrm{mg}$ (EEC via oral). Nesse estudo após 2 anos de tratamento, observou-se que a TRH via transdérmica aumentou significativamente a DMO, mostrando diferenças significativas na coluna lombar e no quadril total (Kim et al., 2014).

Diante dos resultados apresentados, a eficácia e a segurança do uso de géis transdérmicos na veiculação do estrogênio foram promissores, sendo capazes de conter os sintomas menopausais e a manutenção dos níveis hormonais. A via transdérmica continua sendo a alternativa preferida justificada pela vantagem desse sistema na segurança, uma vez que o 
estrogênio administrado por via transdérmica sofre permeação capilar chegando à circulação sistêmica sem passar pelo metabolismo e eliminação gastrointestinal e hepática de primeira passagem. Isso evita a influência na proteína $\mathrm{C}$ reativa, metaloproteinase-9 da matriz e globulina de ligação ao hormônio sexual, sendo uma alternativa segura para mulheres pósmenopausais com risco para a ativação desses fatores e riscos tromboembólicos venosos associados (Kim et al., 2019). A veiculação de estrogênio pela via transdérmica foi seguro, e quando comparado com a via oral, pode reduzir os eventos tromboembólicos venosos e os riscos de AVC. Com relação ao impacto estrogênico na manutenção óssea, houve um aumentou da DMO, que foi comparável ao efeito do estrogênio por via oral. Sendo assim, a via transdérmica para a administração de estradiol veiculado em gel, se mostrou ser a alternativa segura, tolerável e eficaz para a TRH, com a diminuição dos sintomas associados ao climatério.

Os achados para a avaliação da eficácia da TRH transdérmica com gel de estrogênio são promissoras e apontam para uma melhora significativa dos sintomas clínicos vasomotores menopausais

\section{Progesterona}

Os progestágenos são comumente prescritos para mulheres pós-menopáusicas, não apenas para a proteção do endométrio quando usados com estrogênio, mas também quando usado sozinho para o tratamento de hiperplasia endometrial, alívio dos sintomas da menopausa e conservação óssea. Os progestágenos usados para terapia hormonal incluem o progestágeno natural, progesterona e vários progestágenos sintéticos (Stanczyk, 2014). Eles estão disponíveis em diferentes doses e podem ser administrados por via oral e/ou parenteral, em vista disso, a principal via de administração da progesterona natural é a injeção intramuscular devido à sua baixíssima biodisponibilidade via oral. No entanto, uma variedade de progestágenos (progesterona sintética) com ações farmacológicas mais potentes do que a progesterona natural foram desenvolvidos, e muitas formulações orais contendo progesterona tornaram-se comercialmente disponíveis. Portanto, os resultados dos estudos com preparações tópicas de progesterona são muito discutidos quanto à eficácia esperada do efeito protetor endometrial nas formulações, e também quanto à segurança do uso em longo prazo em relação a reações adversas graves, como aumento do risco de câncer de endométrio em pacientes que ainda têm útero (Du et al., 2013).

Nesta revisão não foram encontrados artigos recentes na literatura que avaliassem clinicamente o uso de géis transdérmicos na veiculação do hormônio progesterona. Em vista disso, o parâmetro de corte para os artigos selecionados foram estudos que avaliassem a eficácia e a segurança de formulações tópicas incluindo creme e géis de aplicação local e sistêmica em ensaios clínicos com mulheres menopausais condicionadas a TRH.

Du e colaboradores (2013) realizaram um ensaio clínico prospectivo, randomizado e cruzado com 10 mulheres na pós-menopausa que continham o útero intacto para investigar a distribuição e as respectivas taxas de absorção da progesterona no sangue venoso total, soro venoso, sangue capilar da ponta do dedo e saliva após a aplicação tópica em formulações de creme e gel. O gel consiste em $80 \mathrm{mg}$ de progesterona. Os resultados demonstraram que os valores de progesterona após a aplicação tópica em formulações de creme e gel estão bem abaixo da faixa considerada lútea $(5-25 \mathrm{ng} / \mathrm{mL})$, permanecendo abaixo de $1 \mathrm{ng} / \mathrm{mL}$, com o nível máximo médio de apenas $0,71 \mathrm{ng} / \mathrm{mL}$, indicando que não são capazes de conter a estimulação estrogênica dos tecidos-alvo (Du et al., 2013). Dessa forma, os níveis séricos de progesterona alcançados com o gel foram ligeiramente inferiores aos obtidos com o creme, o que provavelmente são atribuíveis a diferenças na cinética de absorção. Sendo assim, foram encontrados níveis de progesterona no sangue venoso total em mulheres tanto após a aplicação de creme quanto de gel substancialmente menores do que os níveis de progesterona sérica venosa correspondente aos valores medianos da área sob a curva (ASC) de 24 h, de 7,51 e 4,41 ng.h.mL-1, em comparação com 12,39 e 8,32 ng.h.mL-1, respectivamente. Diante dos valores suprafisiológicos de progesterona, os resultados mostraram uma ineficácia da formulação em atingir os 
níveis séricos adequados de progesterona para a proteção do endométrio, sendo necessário um ajuste da formulação e a realização de ensaios clínicos que avaliem também a segurança do uso dos sistemas de géis (Du et al., 2013).

Brkic (2016) realizaram um estudo com 50 mulheres utilizando gel de progesterona para o tratamento da doença fibrocística da mama, onde foi possível diminuir significativamente o número de cistos. Elas foram tratadas com terapia local de gel progesterona (progesterona-1 g natural micronizada), a partir de $16^{\circ}$ a $26^{\circ}$ dia do ciclo durante a fase lútea, com auxílio de um aplicador contendo 0,025 $\mathrm{g}$ de progesterona, em ambas as mamas, duas vezes ao dia, por um período de 6 meses. Durante a terapia com gel de progesterona local para a mastalgia e mastodinia, foi observado que o tamanho médio e número de cistos tiveram seu tamanho reduzido e desapareceu. O objetivo deste estudo foi investigar as mudanças dinâmicas de estrogênio e progesterona durante a fase lútea, sendo o fator etiológico mais importante para a doença fibrocística da mama, sendo assim, o tratamento com o gel de progesterona local pode evitar muitas remoções cirúrgicas desnecessárias de cistos. Além da redução do número de cistos em mulheres tratadas, os resultados indicaram através dos exames de ultrassom da mama e análises hormonais nos períodos de coleta que a mastalgia e a mastodínia diminuíram significativamente durante a terapia com gel local (Brkic et al., 2016)

Porém, apesar da compreensão da etiologia da doença fibrocística da mama, a ação do gel ainda é controversa nas mamas. No entanto Du e colaboradores (2013) e Brkic (2016) mostraram que a concentração dos hormônios nos tecidos é maior do que na circulação, sendo assim, a relação entre os hormônios veiculados estava ainda mais alta, com riscos de superdosagem. Portanto, não é possível avaliar a eficácia dessas preparações transdérmicas apenas por dosagens séricas dos níveis hormonais, a ação local do gel não é detectada na circulação sanguínea, sendo necessários estudos por meio de ensaios clínicos que avaliem seu mecanismo de ação, e o seu perfil de eficácia através de dados farmacocinéticos e exames clínicos que contemplem o seu efeito protetivo do endométrio pela TRH.

Nesse sentido, a via transdérmica é utilizada justamente para se evitar os efeitos adversos causados pelas ações da biologia molecular da progestina, e o mecanismo de primeira passagem via oral através do metabolismo pelo fígado. Porém a distribuição da progesterona não apresentou o efeito esperado, seus níveis na circulação sanguínea foram muito baixos o que levou a conclusão de que a TRH pela via transdérmica não é eficaz, não fornecendo proteção endometrial, em contrapartida seus efeitos precisam ser mais bem avaliados para entender o mecanismo da sua distribuição entre a circulação sanguínea e os tecidos periféricos. Dessa maneira, os dados indicam que a TRH com a progesterona transdérmica, não é capaz de atingir os níveis fisiológicos ideais. Além disso, uma formulação totalmente satisfatória de progesterona ainda não foi desenvolvida, uma vez que a progesterona é conhecida por ser metabolizada na pele por uma 5- $\alpha$-redutase, sendo assim, necessária uma taxa de permeação transdérmica extremamente alta e sustentada para fornecer quantidades terapeuticamente eficazes de progesterona através da pele para a corrente sanguínea para obter ações sistêmicas (Matsui et al., 2015).

Stute (2016) realizou uma revisão sistemática na literatura sobre a progesterona micronizada, em diferentes formas de administração incluindo a via transdérmica, para proteção endometrial, e a conclusão dos especialistas foi que a progesterona transdérmica não oferece proteção endometrial. Os resultados encontrados indicaram que a progesterona micronizada oral fornece proteção endometrial se aplicada sequencialmente por 12-14 dias/mês a $200 \mathrm{mg} / \mathrm{dia}$ por até 5 anos; ao passo que a progesterona micronizada vaginal pode fornecer proteção endometrial se aplicada sequencialmente por 10 dias/mês a $4 \%$ (45 $\mathrm{mg} / \mathrm{dia}$ ) ou em dias alternados a $100 \mathrm{mg} /$ dia por até 3-5 anos (uso off-label); e a progesterona micronizada transdérmica não fornece proteção endometrial. As diretrizes internacionais atuais sobre a TRH recomendam combinar um progestágeno ao usar a terapia de estrogênio em mulheres na peri e pós-menopausa com um útero intacto para proteção endometrial. No entanto, a segurança endometrial em longo prazo após a adição sequencial de progestogênio pode ser reduzida, uma vez que a combinação de estrogênios com progesterona micronizada está associada a um risco aumentado de câncer endometrial se usada por mais de 5 anos. A progesterona micronizada sistêmica está disponível em diferentes dosagens e vias de 
administração, porém, não especificam o tipo de progestagênio, dosagem, via de administração e duração do uso seguro (Stute et al., 2016). Diante disso, os resultados apontam que a progesterona transdérmica não é eficaz, não oferece proteção endometrial, como também não há uma recomendação de qual seria o uso entre as inúmeras formas e vias de administração, porém a segurança em longo prazo para qualquer que seja a via de administração deve ser avaliada em relação aos riscos de câncer endometrial após o uso prolongado.

A pouca quantidade de trabalhos encontrados na literatura envolvendo géis transdérmicos na veiculação da progesterona pode ser explicada pelo número de trabalhos que demonstraram a ineficácia tanto do gel quanto do creme no reestabelecimento dos níveis de progesterona séricos. Conforme discutido, os resultados para essa baixa distribuição da progesterona na circulação sanguínea pode ser resultado da sua metabolização na pele pela 5 - $\alpha$-redutase, o que dificulta a sua permeação transdérmica, como também devem ser investigados os mecanismos da sua distribuição, uma vez que apresentou maior atividade local com acumulação nos tecidos periféricos indicando a não permeação. Diante disso, são necessários mais estudos que avaliem os mecanismos de permeação e distribuição da progesterona, como também os efeitos do seu uso em longo prazo relacionados aos riscos de desenvolvimento de câncer endometrial.

\section{Conclusão}

A revisão da literatura foi realizada para os três hormônios esteroides sexuais, estrogênio, progesterona e testosterona. Diante das limitações encontradas referentes ao número de trabalhos com a progesterona dentro das condições de corte estabelecidas, os resultados obtidos para a segurança e eficácia de géis pela via transdérmica ainda é um desafio, como neste caso da progesterona em que não apresentou permeação através da pele. Apesar disso, o estrogênio e a testosterona se mostraram eficazes, pela via transdérmica, através dos registros da melhora na função sexual e qualidade de vida, e reestabelecimento dos níveis hormonais eugonadais. Porém, a segurança da testosterona não pode ser garantida devido à instabilidade dos picos hormonais presentes na circulação sistêmica, apesar dos eventos adversos serem considerados leves, a segurança em longo prazo precisa ser mais bem avaliada. Deste modo, a TRH mais segura e eficaz utilizando gel transdémico foi para a veiculação do hormônio estrogênio, enquanto que a testosterona foi eficaz, mas não pode ter a sua segurança garantida, e a progesterona não apresentou nem eficácia e nem segurança. Todavia, a avaliação do uso da TRH com géis transdérmicos deve ser ponderada de acordo com a condição clínica de cada paciente, uma vez que o uso da via transdérmica continua sendo a via mais prática e de maior adesão pelos pacientes submetidos à terapia.

Dessa forma, é necessário realizar estudos em longo prazo que acompanhem as consequências da TRH com a testosterona, mantendo o ajuste de doses e exames periódicos para a garantia da segurança e a efetividade do tratamento. Também realizar estudos que avaliem clinicamente o uso de géis transdérmicos na veiculação do hormônio progesterona.

\section{Referências}

Allen, JR. L. V., Popovich, N.G., \& Ansel, H.C. (2007). Formas farmacêuticas e sistemas de liberação de fármacos. (8a ed.), Artmed.

Archer, D. F., Pickar, J. H., MacAllister, D. C., \& Warren, M. P. (2012). Transdermal estradiol gel for the treatment of symptomatic postmenopausal women. Menopause, 19(6), 622-629. https://doi.org/10.1097/gme.0b013e31823b8867.

Belkoff, L., Brock, G., Carrara, D., Neijber, A., Ando, M., \& Mitchel, J. (2018). Efficacy and safety of testosterone replacement gel for treating hypogonadism in men: Phase III open-label studies. Andrologia, 50(1), 1-7. https://doi.org/10.1111/and.12801.

Brkic, M., Vujovic, S., Ivanisevic, M. F., Ivovic, M., Gajic, M. T., Marina, L., Barac, M., Barac, B., Djogo, A., Malesevic, G., \& Franic, D. (2016). The Influence of Progesterone Gel Therapy in the Treatment of Fibrocystic Breast Disease. April, 334-341.

Canonico, M., Carcaillon, L., Plu-Bureau, G., Oger, E., Singh-Manoux, A., Tubert-Bitter, P., Elbaz, A., \& Scarabin, P. Y. (2016). Postmenopausal hormone therapy and risk of stroke: Impact of the route of estrogen administration and type of progestogen. Stroke, 47(7), 1734-1741. https://doi.org/10.1161/STROKEAHA.116.013052 
Carniél, M. A. (2013). Formulações tópicas magistrais para terapia de reposição hormonal em mulheres no período pós-menopausa e a aplicação da atenção farmacêutica. Trabalho de Conclusão de Curso. Faculdade de farmácia, Universidade Federal do Rio Grande do Sul. https://www.lume.ufrgs.br/handle/10183/144024.

Cauley, J. A. (2015). Estrogen and bone health in men and women. Steroids, 99(Part A), 11-15. https://doi.org/10.1016/j.steroids.2014.12.010.

Cunha, I. V. N. (2020). Uma revisão dos principais sistemas transdérmicos de testosterona utilizados em terapias de reposição hormonal. Trabalho de Conclusão de Curso. Departamento de ciências farmacêuticas, Universidade Federal de Santa Catarina. https://repositorio.ufsc.br/handle/123456789/209575.

Dobs, A. S., Mcgettigan, J., Norwood, P., Howell, J., Waldie, E., \& Chen, Y. (2012). A novel testosterone $2 \%$ gel for the treatment of hypogonadal males. Journal of Andrology, 33(4), 601-607. https://doi.org/10.2164/jandrol.111.014308.

Du, J. Y., Sanchez, P., Kim, L., Azen, C. G., Zava, D. T., \& Stanczyk, F. Z. (2013). Percutaneous progesterone delivery via cream or gel application in postmenopausal women: a randomized cross-over study of progesterone levels in serum, whole blood, saliva, and capillary blood. Menopause (New York, N.Y.), 20(11), 1169-1175. https://doi.org/10.1097/GME.0b013e31828d39a2.

Efros, M., Carrara, D., \& Neijber, A. (2016). The efficacy, bioavailability and safety of a novel hydroalcoholic testosterone gel $2 \%$ in hypogonadal men: results from phase II open-label studies. Andrologia, 48(6), 637-645. https://doi.org/10.1111/and.12493.

Farwell, W. R., Jette, A. M., Ph, D., Eder, R., Tennstedt, S., Ph, D., Mazer, N. A., Ph, D., Miciek, R., Krasnoff, J., Ph, D., Elmi, A., Knapp, P. E., Brooks, B., Appleman, E., Aggarwal, S., Bhasin, G., Hede-brierley, L., Bhatia, A., \& Bhasin, S. (2010). New england journal. $109-122$.

Gomes, F. P., \& Boldo, M. E. (2020). Investigation of in vitro permeation and release assays of semi-solid topical formulations to standardization of quality requirements on a pharmaceutical industry. Research, Society and Development, 9 (11), e59491110210. https://rsdjournal.org/index.php/rsd/article/view/10210.

Hadgraft, J., \& Lane, M. E. (2015). Transdermal delivery of testosterone. European Journal of Pharmaceutics and Biopharmaceutics, 92 (February), $42-48$. https://doi.org/10.1016/j.ejpb.2015.02.015.

Johansen, N., Lindén Hirschberg, A., \& Moen, M. H. (2020). The role of testosterone in menopausal hormone treatment. What is the evidence? Acta Obstetricia et Gynecologica Scandinavica, 99(8), 966-969. https://doi.org/10.1111/aogs.13819.

Kamal, N. S., Alayoubia, A., Elfakhria, K. H., Ibrahimb, S., Seggel, M., Ashraf, M., Zidan, A. (2020). Effects of formulation variables on the in vitro performance of testosterone transdermal gel. International Journal of Pharmaceutics, 590, 119951 -119965. https://doi.org/10.1016/j.ijpharm.2020.119951

Kaufman, J. M., Miller, M. G., Garwin, J. L., Fitzpatrick, S., Mcwhirter, C., \& Brennan, J. J. (2011). Efficacy and Safety Study of 1.62\% Testosterone Gel for the Treatment of Hypogonadal Men. Journal of Sexual Medicine, 8(7), 2079-2089. https://doi.org/10.1111/j.1743-6109.2011.02265.x.

Kim, H. J., Oh, Y. K., Lee, J. S., Lee, D.-Y., Choi, D., \& Yoon, B.-K. (2014). Effect of Transdermal Estrogen Therapy on Bone Mineral Density in Postmenopausal Korean Women. Journal of Menopausal Medicine, 20(3), 111. https://doi.org/10.6118/jmm.2014.20.3.111.

Kim, H. K., Jeon, S. H., Ryu, K.-J., Kim, T., \& Park, H. (2019). Comparison of the Efficacy of Tibolone and Transdermal Estrogen in Treating Menopausal Symptoms in Postmenopausal Women. Journal of Menopausal Medicine, 25(3), 123. https://doi.org/10.6118/jmm.19205

Kumar, A., Banerjee, A., Singh, D., Thakur, G., Kasarpalkar, N., Gavali, S., Gadkar, S., Madan, T., Mahale, S. D., Balasinor, N. H., \& Sachdeva, G. (2018). Estradiol : A Steroid with Multiple Facets I Estrogens in Normal Physiology Estrogen synthesis. Hormones Metabolism Research, 50(5), 359-374.

Lima, J. V. de O., Cavalcante, L. C. da S.; Lima, D. C. dos S.; Barbosa, M. C. N. A.; Silva, I. P.; Luz, A. L. R.; Costa, M. H. de A. da; Nunes, M. H.; Batista, M. de K. P.; Júnior, J. L. P.; Cavalcante, G. L. (2020). Risk-benefit of hormone replacement therapy in menopausal women. Research, Society and Development, 9, 5, e07952283. https://rsdjournal.org/index.php/rsd/article/view/2283.

L'Hermite, M. (2017). Bioidentical menopausal hormone therapy: registered hormones (non-oral estradiol \pm progesterone) are optimal. Climacteric, 20(4), 331-338. https://doi.org/10.1080/13697137.2017.1291607.

Matsui, R., Ueda, O., Uchida, S., \& Namiki, N. (2015). Transdermal absorption of natural progesterone from alcoholic gel formulations with hydrophilic surfactant. Drug Development and Industrial Pharmacy, 41(6), 1026-1029. https://doi.org/10.3109/03639045.2014.925915.

Melo, I. A. C.; Sousa, P. E. L.; Lemos-Jordão, A. J. J. M. Estrogen and progesterone in postmenopausal hormone therapy: a systematic review of influence in cognitive domain. Research, Society and Development, 10 (12), e64101220075, 2021. https://rsdjournal.org/index.php/rsd/article/view/20075.

Mizunuma, H. (2011). Clinical usefulness of a low-dose maintenance therapy with transdermal estradiol gel in Japanese women with estrogen deficiency symptoms. Climacteric, 14(5), 581-589. https://doi.org/10.3109/13697137.2011.570388.

Mohamad, N. (2016). Uma revisão concisa da testosterona e saúde óssea. 1317-1324.

Pardini, D. (2014). Terapia de reposição hormonal na menopausa Hormone replacement therapy in menopause. Arq Bras Endocrinol Metab, 58(2), 10. 172181. https://doi.org/10.1590/0004-2730000003044.

Piette, P. (2018). The history of natural progesterone, the never-ending story. Climacteric, 21(4), 308-314. https://doi.org/10.1080/13697137.2018.1462792

Polonini, H. C., Brandão, M. A. F., \& Raposo, N. R. B. (2011). a Terapia De Reposição Hormonal E a Saúde Da Mulher No Climatério: Riscos E Benefícios. Revista de APS, 14(3), 354-361.

Renoux, C., Dell'aniello, S., \& Suissa, S. (2010). Hormone replacement therapy and the risk of venous thromboembolism: A population-based study. Journal of Thrombosis and Haemostasis, 8(5), 979-986. https://doi.org/10.1111/j.1538-7836.2010.03839.x. 
Research, Society and Development, v. 10, n. 16, e428101623891, 2021

(CC BY 4.0) | ISSN 2525-3409 | DOI: http://dx.doi.org/10.33448/rsd-v10i16.23891

Sansone, A., Sansone, M., Selleri, R., Schiavo, A., Gianfrilli, D., Pozza, C., Zitzmann, M., Lenzi, A., \& Romanelli, F. (2019). Monitoring testosterone replacement therapy with transdermal gel: when and how? Journal of Endocrinological Investigation, 42(12), 1491-1496. https://doi.org/10.1007/s40618-01901082-x.

Schulster, M., Bernie, A. M., \& Ramasamy, R. (2016). The role of estradiol in male reproductive function. Asian Journal of Andrology, 18(3), 435-440. https://doi.org/10.4103/1008-682X.173932.

Singh, D., Pradhan, M., Nag, M., \& Singh, M. R. (2015). Vesicular system: Versatile carrier for transdermal delivery of bioactives. Artificial Cells, Nanomedicine and Biotechnology, 43(4), 282-290. https://doi.org/10.3109/21691401.2014.883401.

Stanczyk, F. Z. (2014). Treatment of postmenopausal women with topical progesterone creams and gels: Are they effective? Climacteric, 17(Suppl 2), 8-11. https://doi.org/10.3109/13697137.2014.944496.

Stute, P., Neulen, J., \& Wildt, L. (2016). The impact of micronized progesterone on the endometrium: a systematic review. 7137. https://doi.org/10.1080/13697137.2016.1187123.

Taraborrelli, S. (2015). Physiology, production and action of progesterone. Acta Obstetricia et Gynecologica Scandinavica, $94,8-16$. https://doi.org/10.1111/aogs.12771. 